\title{
Focusing Properties of a Modified Retarding Structure for Linear Electron Accelerators
}

\author{
Vladimir Kuz'michShilov, Aleksandr Nikolaevich Filatov, Aleksandr Evgen'evich Novozhilov \\ National Research Nuclear University MEPhI (Moscow Engineering Physics Institute), Kashirskoye Shosse 31, Moscow, \\ 115409, Russian Federation
}

\begin{abstract}
Article Info
Article history:

Received Jun 9, 2016

Revised Mar 10, 2017

Accepted Mar 24, 2017

Keyword:

Acceptance structures

Beam emittance

Biperiodic retarding system

Drift sleeve

Electromagnetic field of the cell

Electron linear accelerator

Particle dynamics

Standing wave

The drift channel aperture

ABSTRACT

When using accelerators in industry and medicine, important are the dimensions of the device used, especially the radial ones. In the linear electron accelerators based on a biperiodic retarding structure, which operates in the standing wave mode, there is a possibility to provide focusing of the accelerated particles with the help of high-frequency fields without the use of external focusing elements. In the accelerating cell, due to the presence of the far protruding drift sleeves, the electric field lines become strongly curved, which leads to the appearance in the regions adjacent to these sleeves of a substantial in magnitude radial component of the electric field. The particles entering the accelerating gap experience the action of a force directed toward the axis of the system, and at the exit, of a force directed away from the axis. Under certain conditions, alternation of the focusing and defocusing fields can lead to a general focusing effect.In the paper we study the focusing properties of a modified biperiodic structure with standing wave. The main attention is paid to the possibility of using the focusing properties of the electromagnetic accelerating field for guiding the electron beam through the aperture of the accelerating system, which will lead to a significant reduction in the accelerator sizes. The proposed method can be applied in the calculation and design of linear electron accelerators.
\end{abstract}

Copyright () 2017 Institute of Advanced Engineering and Science. All rights reserved.

\author{
Corresponding Author: \\ Vladimir Kuz'mich Shilov, \\ Department of Automation and Electronics, \\ National Research Nuclear University MEPhI (Moscow Engineering Physics Institute), \\ Kashirskoye shosse 31, Moscow, 115409, Russian Federation. \\ Email: VKShilov@mephi.ru
}

\section{INTRODUCTION}

The interest in the use of linear electron accelerators (LEA) in industry and medicine can be attributed to a number of their advantages. An analysis of the output characteristics of the deceleration radiation in defectoscopy and radiation therapy [1] shows that a large part of the radiation problems in these fields can be solved with the help of LEA for the energy of $4-6 \mathrm{MeV}$ and the pulse current of $50-150 \mathrm{~mA}$. In addition, the accelerators used in defectoscopy and medicine should be compact and have small longitudinal and transverse dimensions. Undoubtedly, reducing the sizes and weight of the radiation installations is an important and urgent task. From the very beginning, this problem was solved by the transition to the three-centimeter wavelength range. Currently, the reduction of the longitudinal dimensions can be achieved by switching to the standing wave mode [2]. In this case, as the retarding systems in the LEA with standing wave, the biperiodic retarding structures (BRS) [3] are used, which are characterized by high values of the specific shunt resistances and the gradient of the accelerating field.

As is known, in the traveling wave LEA, the particle gains energy only under the condition of the synchronous movement with the accelerating wave. Due to the relatively low shunt resistance of the accelerating structures operating in the traveling wave mode, the accelerated particle should be at the 
maximum of the accelerating field. The constant influence on the particle from the radial component of electric field which deflects it from the axis is the reason that the external focusing elements, especially in the beginning of acceleration, have become an integral part of the design of the traveling wave accelerators. This requires additional energy costs and makes the accelerator design more cumbersome.

A special place among the BRS that are widely used as accelerating systems for the linear electron accelerators for low energy with a standing wave is occupied by the structure [4], using which it is possible to achieve a high rate of acceleration. Concerning this structure,there is a pressing issue of providing radial focusing of the accelerated particles, since the outer diameter of the structure due to lateral connection cells extending from the four sides of the accelerating cells arranged on one axis is approximately twice the diameter of the accelerating structures with the axial connection cells, and the use of conventional focusing devices is therefore associated with a considerable increase in the transverse dimension which is already large. Moreover, the mass of the accelerator's transmitter unit increases, and there arises the need to introduce into the installation the power supplies for the focusing elements.

\section{METHODS}

\subsection{General Statements}

BRS in the LEA, working in the standing wave mode, can be considered as a chain of accelerating cavities, arranged on a single axis and linked by the connection cells [5]. Since the connection cells are free from electromagnetic fields and not involved in the particle acceleration, they may be placed outside of the accelerating structure. The oscillations of electromagnetic fields in the adjacent accelerating cavities are different in phase by $\pi$; therefore, the particle must cover the distance between the centers of adjacent accelerating cavities during the time equal to half the period of electromagnetic oscillations. Only in this way the synchronization is achieved between the particle and the accelerating field.

If we consider, ata fixed moment of time, the distribution function of the electromagnetic field intensity along the axis of the LEA with standing wave, then we can see that this function consists of alternating pulses of different polarity with a spatial period equal to the wavelength of the generator.

Under some well-known conditions [6], this distribution function can be expanded in a Fourier series, and the standing wave field can be represented by a sum of harmonics. The particle interacts effectively only with the harmonic, the phase velocity of which equals the velocity of the particle motion. Consequently, the longitudinal dynamics of the particles in the LEA with standing wave can be studied by the methods used in the traveling wave accelerators.

When considering the radial motion of the particles,it is necessary to note the fact that the longitudinal and radial motions are closely connected and their equations must be solved together. In the LEA with standing wave on the basis of BRS, we can note some specific characteristics in the calculation of the radial motion of the particles. In the case of the accelerating cavities of complex shape optimized with respect to shunt resistance, there are no analytical expressions for the components of electromagnetic field. The distributions of the electromagnetic field components with respect to the longitudinal coordinate and the radius, obtained with the help of numerical methods, must be given in the form of tables over the entire aperture of the drift channel. The presence in the accelerating cells of the protruding drift sleeves is the cause of strong curvature of the field lines of the electric field, which leads to the appearance of the significant in magnitude transverse electric fieldat the place of the largest curvature. Besides, the drift sleeves shield a certain part of the drift channel from the electromagnetic field, resulting in the appearance in the accelerating structure of the periodically repeated segments free from electromagnetic field.

\subsection{Dynamics of the Particles}

The most promising in the LEA with standing wave is the implementation of focusing, for which the focusing action of the electromagnetic field itself is used for guiding the beam [7]. As mentioned above, it becomes possible due to the appearance in the BRS cells of the radial component of electric field. Using the focusing properties of the ownhigh-frequency (HF) field of the BRS significantly reduces the transversal sizes of the accelerating section and lightens the construction of the accelerator as a whole. Furthermore, there is no longer the need for cumbersome power sources for the external focusing devices creating additional difficulties in the use of accelerators, which is particularly important when used in geology, medicine and defectoscopy.

The dynamics of the beam in the linear accelerator is determined by the movement of each particle contained in the beam under the action of electric and magnetic fields. If we assume that the particle charge is pointlike and its own magnetic moment is absent [8], then the equation of motion of the individual particle is 
described by the following expression

$$
\frac{d}{d t}(m \boldsymbol{v})=q \boldsymbol{E}+q \mu_{0}[\boldsymbol{v} \boldsymbol{H}]
$$

where $t$ is time, $q$ and $m$ are the charge and mass of the particle, $v$ is the velocity of the particle, $\mu_{0}$ is the magnetic constant, $\boldsymbol{E}$ and $\boldsymbol{H}$ are high-frequency electromagnetic, static electrical or magnetic focusing fields, or the fields created by other beam particles.

Because ultimately we are interested in the trajectory of the beam particles in the accelerator, the equation (1) should be converted to a form convenient for the study of the particle dynamics, that is, we should express the acceleration of the particle through its speed and the intensity of the electric and magnetic fields. Since the velocities of the particles in the accelerator are close to the speed of light, then, using the well-known formulas of relativistic mechanics [9], we can get

$$
\frac{d v}{d t}=\frac{q}{m_{0}} \sqrt{1-\frac{v^{2}}{c^{2}}}\left\{\boldsymbol{E}+\mu_{0}[\boldsymbol{v H}]-\frac{1}{c^{2}} \boldsymbol{v}(\boldsymbol{v} \boldsymbol{E})\right\}
$$

In general, the vectors $\boldsymbol{E}$ and $\boldsymbol{H}$ depend on the coordinates and time; in the particular case, when the time dependence is absent (the case of the static electric and magnetic fields), the formula retains its form.

Let us project the resulting vector equation (2) on the coordinate axes. In our case, the most convenient is to use a cylindrical coordinate system $(\rho, \varphi, z)$, since the drift channel and the electromagnetic field $E_{010}$ for the lowesttype of oscillations have axial symmetry. Let us move to the dimensionless coordinates $\eta=\frac{\rho}{\lambda}, \eta=\frac{\rho}{\lambda}$, the dimensionless time $\tau=\frac{c t}{\lambda}$, the relative velocities with respect to coordinates $\beta=\frac{d \xi}{d \tau}, u=\frac{d \eta}{d \tau}, \alpha=\eta \frac{d \varphi}{d \tau}$ and the dimensionless components of the electrical and magnetic fields, which can be easily reduced to a dimensionless form using the expressions:

$$
\begin{aligned}
& A_{i}(\eta, \varphi, \xi, \tau)=\frac{q \lambda}{m_{0} c^{2}} E_{i}(\eta, \varphi, \xi, \tau), \\
& B_{i}(\eta, \varphi, \xi, \tau)=\frac{q \lambda}{m_{0} c^{2}} Z_{0} H_{i}(\eta, \varphi, \xi, \tau)
\end{aligned}
$$

where $\lambda$ is the wavelength of the generator, $i=\eta, \varphi, \xi$.

As a result, the equation of motion of the charged particle can be represented by a system of equations in dimensionless quantities

$$
\begin{aligned}
& \frac{d^{2} \xi}{d \tau^{2}}=\frac{1}{\gamma}\left\{A_{\xi 0}(\eta, \xi) \cos (2 \pi \tau)+u B_{\varphi 0}(\eta, \xi) \sin (2 \pi \tau)-\alpha B_{\eta}(\eta, \xi)-\beta\left[u A_{\eta 0}(\eta, \xi) \cos (2 \pi \tau)+\beta A_{\xi 0}(\eta, \xi) \cos (2 \pi \tau)\right]\right\} \\
& \begin{aligned}
\frac{d^{2} \eta}{d \tau^{2}}= & \frac{1}{\gamma}\left\{A_{\eta 0}(\eta, \xi) \cos (2 \pi \tau)+\alpha B_{\xi}(\eta, \xi)-\beta B_{\varphi 0}(\eta, \xi) \sin (2 \pi \tau)-u\left[u A_{\eta 0}(\eta, \xi) \cos (2 \pi \tau)+\beta A_{\xi 0}(\eta, \xi) \cos (2 \pi \tau)\right]\right\} \\
& +\frac{\alpha^{2}}{\eta},(3)
\end{aligned} \\
& \begin{aligned}
\frac{d \alpha}{d \tau}= & \frac{1}{\gamma}\left\{\beta B_{\eta}(\eta, \xi)-u B_{\xi}(\eta, \xi)-\alpha\left[u A_{\eta 0}(\eta, \xi) \cos (2 \pi \tau)+\beta A_{\xi 0}(\eta, \xi) \cos (2 \pi \tau)\right]\right\}-\frac{\alpha u}{\eta}
\end{aligned}
\end{aligned}
$$

In the latter system of equations, $\gamma=\frac{1}{\sqrt{1-\beta^{2}-u^{2}-\alpha^{2}}}$ is the energy of the particle in the units of the rest energy.

The resulting system of equations (3) has been taken as the basis for the study of the motion of particles in the accelerators with standing wave. Due to the fact that it is impossible to present analytically the electromagnetic field in the accelerating cavity of a complex shape, the corresponding components of the field, included in the right-hand side, should be given as a table. The external magnetic field can be of any type and is also given by a table.

\section{DISCUSSION AND RESULTS}

Let us analyze the effect of high frequency (HF) focusing, which in the considered case has a number of features. To obtain a clear physical picture, we use a simplified mathematical model of accelerating cells proposed in [10], with the only difference that its longitudinal dimension will be two times less. After calculations, we conclude that when the accelerating field varies with time according to the cosine law, then, in this case, all the regions of the initial phases of the electron flying in, for which there are

Focusing Properties of a Modified Retarding Structure for Linear Electron ... (Vladimir Kuz'mich Shilov) 
realized the conditions of accelerating and focusingby the corresponding components of the HF field, will be shifted by $\pi / 4$ as compared to the analogous regions for the traditional BRS.

Therefore, in the design of the accelerating cells with the drift sleeves or without them in the form of conventional cylindrical accelerating cavities, the phase regions of simultaneous acceleration and stable radial motion for the first type of cells will be

$$
-\frac{\pi}{4} \pm 2 \pi k<\varphi_{0}<\frac{\pi}{4} \pm 2 \pi k
$$

and for the cells of the second type

$$
-\frac{3 \pi}{4} \pm 2 \pi k<\varphi_{0}<-\frac{\pi}{4} \pm 2 \pi k
$$

where $k=0,1,2$...It may be noted that the extent of the phase regions is, as before, $\frac{\pi}{2}$.

In the paper, we consider the design and study the characteristics of a modified accelerating structure of this type with the improved focusing properties. This is achieved by the design of the accelerating cells with one drift sleeve and the drift opening of conical shape, which changes the spatial configuration of the HF fields in the system and results in a change of boundaries of the corresponding phase regions of acceleration and focusing. Amodified BRS is shown in Figure 1.
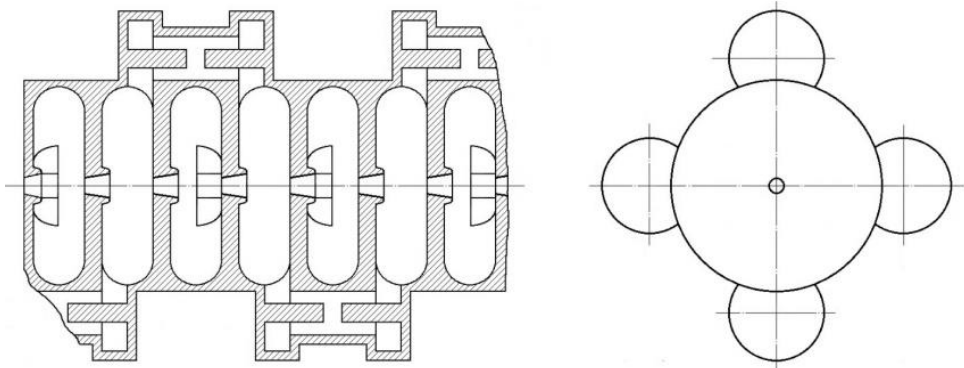

Figure1. Modified BRS with a high acceleration rate.

In our case, without a serious damage to the final result, we can neglect the change in the shape of the distribution of the azimuthal magnetic and longitudinal electric fields, taking into account only a significant change in the radial component of the electric field. Then for the electron beam entering the accelerating cellfrom the direction of the drift sleeve, the region of focusingby the radial electric field will coincide with the corresponding region of the traditional BRS, whereas the region of the simultaneous acceleration and focusing will be

$$
-\frac{\pi}{2} \pm 2 \pi k<\varphi_{0}<\frac{\pi}{4} \pm 2 \pi k
$$

Moreover, the focusing zones due to the use of the radial component of electric field and the azimuthal component of magnetic field overlap in the range

$$
-\frac{\pi}{2} \pm 2 \pi k<\varphi_{0}<-\frac{\pi}{4} \pm 2 \pi k
$$

Thus, we achieve a $50 \%$ increase for the sizes of the region of the initial phases of the electron flying in, for which the conditions of simultaneous acceleration and focusing are fulfilled, and, besides, the phase corresponding to the maximum acceleration $(\pi / 4)$ falls within the focusing region. This makes it possible to implement the mode which providesboth the radial focusing of the electron bunches and their optimal acceleration. Such favorable in all respects mode of the installation is unattainable while using BRS with the conventional accelerating cells. 
More complete information on the focusing properties of both high frequency structures could be obtained by the software for integrating the motion equations of charged particles in the structures working in the standing wave mode. The HF fields interacting with the electrons along the entire length of the accelerator were given in tables. We investigated an accelerating system containing 16 identical accelerating cells, the longitudinal dimension of which corresponded to the relative phase velocity equal to 1 , while the intensity of the longitudinal electric field on the axis of the accelerating cells was $180 \mathrm{kV} / \mathrm{cm}$. The phases were measured in the scale where 1 corresponded to $2 \pi$. The particles fell into a structure with the initial radius of $1 \mathrm{~mm}$, with the longitudinal relative velocity $\beta_{z}=0.97$ and the relative radial velocity $\beta_{\rho}=0.01$.

The calculation results indicate that the focusing effect on the accelerated electrons of the modified HF structure is much more significant. Using the concept of acceptance, we have succeeded in carrying out a correct comparison of the focusing properties of the conventional BRS of the considered type and the BRS with a modified profile of accelerating cells.

The acceptance is the maximal possible emittance of the coherent beam that is able to pass through the system. The higher the acceptance, the higher is the carrying capacity of the HF structure. Figure 2 presents the acceptances for various initial phases of the electron flying into the considered systems, where both systems have the same apertures of $10 \mathrm{~cm}$.

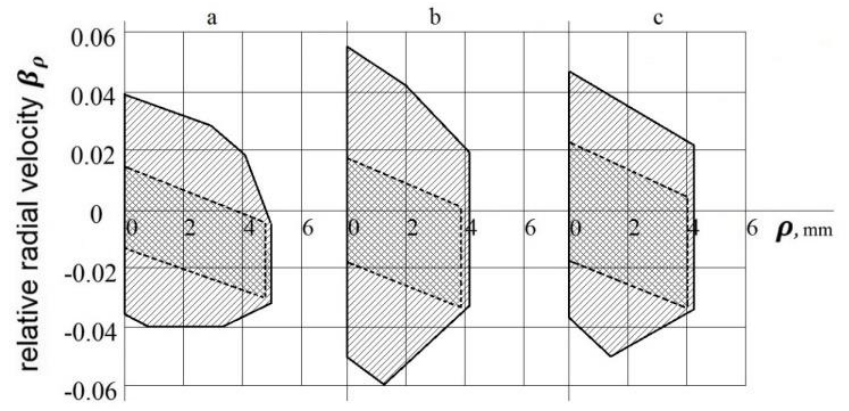

Figure 2. Acceptances for various initial phases of the electrons flying into the accelerating systems: a-the phase $0.7, \mathrm{~b}$-the phase $0.8, \mathrm{c}-$ the phase 0.9 ; the dashed linere presents the acceptances of the conventional BRS, the continuous line, the acceptances of the modified BRS.

As seen from Figure 2, the described change of the form of accelerating cells allows increasing by two times and more the phase area bounded by the acceptances for the entire phase range corresponding to the maximum gain by the particles of the output energy (the phases 0.7-0.9).

The main advantage of the standing-wave LEA with BRS is the possibility of a significant reduction in the length of the accelerating structure to yield the necessary gaining of energy under the same power of the HF generator. Obviously, such a structure should have, first of all, a large value of effective shunt resistance. Among other electrodynamic characteristics of BRS, the connection coefficient between cells, the quality factor, the group velocity, the maximum values of the electric and magnetic fields on the surface of the structure, the electric field overvoltage factor are important.

Accelerators with a large connection coefficient have better stability to changes in the parameters of the accelerated beam, less rigid tolerances for manufacturing the accelerating structure, better stability of operation of the HF generator. In [11], there are presented the results of studies on optimizing the geometry of BRS with internal connection cells with respect to increasing the coupling coefficient to $15 \%$ under an insignificant change in the line effective shunt resistance and other electrodynamic characteristics. The results indicate the promising character of the research in this direction [12-14].

Thus, the proposed modification of the shape of the BRS accelerating cells has a number of indisputable advantages and allows significantly increasing the efficiency of the system in such promising mode as focusing of the accelerated be amby the own HF fieldsof the accelerating structure.

\section{CONCLUSION}

In conclusion, we would like to mention the fact that the calculation results have fully confirmed the conclusions obtained using a simplified mathematical model of the accelerating cell and provide a clear picture of the processes. Therefore, in the future the authors plan to use it for the continuous determination of the focusing properties of various HF structures working in the standing wave mode.

Focusing Properties of a Modified Retarding Structure for Linear Electron ... (Vladimir Kuz'mich Shilov) 
The proposed method of guiding the electron beam in linear accelerators is based on using the focusing properties of the radial component of the electric field. This component appears even for the lowest type of oscillation in the conventional cylindrical accelerating cavities in the region of the drift tubes and, therefore, in the vicinity of the drift channel axis. This effect, arising due to the curvature of the electromagnetic field lines, might be promising also for the proton and ion linear accelerators.

The studies conducted with the help of mathematical modeling have shown the promising nature of this method in the design and construction of the LEA without the use of external focusing devices. This will allow greatly reducing the dimensions and weight of the accelerator itself and enhancing its effectiveness while using in the technological fields of industry and medicine.

Inthe future, we plan to study the impact of changes in the shape the BRS accelerating cavities on the distribution of the electromagnetic field components in the region of drift channel and on the conditions of effective acceleration and radial stability.

\section{ACKNOWLEDGEMENTS}

This work was supported by MEPhI Academic Excellence Project (contract No. 02.a03.21.0005, 27.08.2013).

\section{REFERENCES}

[1] K. Belovintzev, et al., "Izluchatel'nyy kompleks dlya fundamental'nykh i prikladnykh issledovaniy," [Radiation Complex for Fundamental and Applied Research]. In Trudy 14 soveshchaniya po uskoritelyam zaryazhennykh chastits, Protvino [14th conference on charged particles' accelerators, Protvino], Vol. 4, pp. 264-268, 1994.

[2] A.N. Filatov \& V.K. Shilov, "Control of radio-frequency characteristics of linear electron accelerator". Instruments and Experimental Techniques New York, 28(6 pt 1), 1258-1261, 1985.

[3] A.E.Novozhilov, et al., "Calculation of Resonant Frequencies and Electromagnetic Fields in Resonators of Linear Accelerators for Commercial Application, Medicine and Environmental Protection,"Research Journal of Pharmaceutical, Biological and Chemical Sciences, 7(2), 897-905, 2016.

[4] V.A. Vaguine, "Standing Wave High Gradient Accelerator Structure", IEEE Transactions on Nuclear Science, 24(3), 1084-1086, 1977.

[5] A.E. Novozhilov, et al., "Distribution of Accelerating Voltage in Resonator of Linear Electron-Positron Collider", International Journal of Applied Engineering Research, 11(3), 1596-1602, 2016.

[6] M. Vygodskiy, "Spravochnik po vysshey matematike" [Handbook on Higher Mathematics]. Moscow: AST, Astrel', 2006.

[7] Ya. Fainberg, "O vozmozhnosti odnovremennoy radial'noy i fazovoy ustoychivosti v lineynykh uskoritelyakh bez spetsial'nykh fokusiruyushchikh ustroystv," [On thePossibility of Simultaneous Radial andPhase Stability in Linear Accelerators without Special Focusing Devices].In Teoriya i raschet lineynykh uskoriteley [Theory and calculation of linear accelerators].Moscow: Gosatomizdat, 1962.

[8] A.E. Novozhilov, et al., "Problems of measurement of high-frequency fields in linear electron accelerators", Global Journal of Pure and Applied Mathematics, 12(1), 643-655, 2016.

[9] L. Landau \& E. Lifshitz, "Teoreticheskaya fizika: Tom 2. Teoriya polya" [Theoretical Physics: Vol. 2. Field Theory]. Moscow: Science. 1988.

[10] A.N. Filatov \& V.K. Shilov, "RF Focusing in the Standing-Wave Electron Linacs", Soviet physics. Technical physics, 29(2), 163-167, 1984.

[11] E.A. Savin, N.P. Sobenin, "Biperiodic accelerating structure with internal connection cells with an increased connection coefficient", Journal of Technical Physics, 83(5), 141-146, 2013.

[12] N.N.N.A. Malik, M. Esa, S.K.S. Yusof, N.M.A. Latiff "Intelligent Linear Collaborative Beamforming for Multiobjective Radiation Beampattern in Wireless Sensor Networks" TELKOMNIKA Indonesian Journal of Electrical Engineering, 12(10), 7318-7329, 2014.

[13] Suliman Abdel Rahman Abdalla "A Real-Time Implementation of Moving Object Action Recognition System Based on Motion Analysis" Indonesian Journal of Electrical Engineering and Informatics, 5(10), 2017.

[14] Sobhan Dorahaki, "Evaluating the Radiation and Temperature Effect on Photovoltaic Systems", Bulletin of Electrical Engineering and Informatics, 4(1), 1-6, 2015. 


\section{BIOGRAPHIES OF AUTHORS}

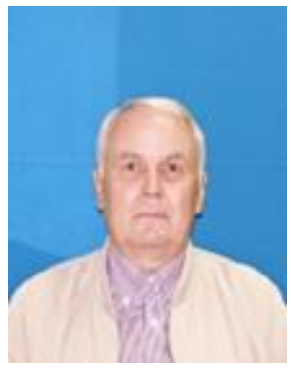

A.N. Filatov \& V.K. Shilov, "RF Focusing in the Standing-Wave Electron Linacs," Soviet physics. Technical physics,29(2), 163-167, 1984

A.N. Filatov \& V.K. Shilov, CONTROL OF RADIO-FREQUENCY CHARACTERISTICS OF LINEAR ELECTRON ACCELERATOR. Instruments and experimental techniques New York, Volume 28, Issue 6 pt 1, November 1985, Pages 1258-1261

Problems of Beam Focusing by Electromagnetic Field in Linear Electron Accelerator with Standing Wave, Based on Biperiodic Structure Novozhilov A.E., Filatov A.N., Shilov V.K.Modern Applied Science; Vol. 9, No. 4; 2015: 160-169 ISSN 1913-1844 E-ISSN 19131852 Pub-lished by Canadian Center of Science and Education DOI:10.5539/mas.v9n4p160

Linear Electron Accelerators Aimed at Environmental Protection Novozhilov A.E., Filatov A.N., Shilov V.K. Biosciences Biotechnology Research Asia, September 2015. Vol. 12(Spl. Edn. 2), p. 261-268 DOI: http://dx.doi.org/10.13050/bbra/2199

Compact Accelerating System for a Multichannel Electron Linear Accelerator Novozhilov A.E., Filatov A.N., Shilov V.K. Research Journal of Pharmaceutical, Biological and Chemical Sciences (ISSN09758585-India-Scopus) July - August 2016 RJPBCS 7(5) Page No. 939-944

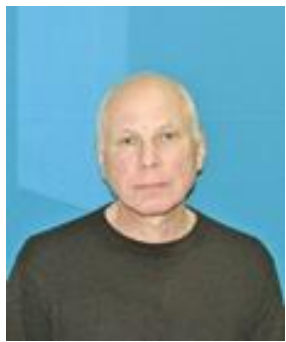

A.N. Filatov \& V.K. Shilov, "RF Focusing in the Standing-Wave Electron Linacs," Soviet physics. Technical physics,29(2), 163-167, 1984

A.N. Filatov \& V.K. Shilov, CONTROL OF RADIO-FREQUENCY CHARACTERISTICS OF LINEAR ELECTRON ACCELERATOR. Instruments and experimental techniques New York, Volume 28, Issue 6 pt 1, November 1985, Pages 1258-1261

Problems of Beam Focusing by Electromagnetic Field in Linear Electron Accelerator with Standing Wave, Based on Biperiodic Structure Novozhilov A.E., Filatov A.N., Shilov V.K.Modern Applied Science; Vol. 9, No. 4; 2015: 160-169 ISSN 1913-1844 E-ISSN 19131852 Pub-lished by Canadian Center of Science and Education DOI:10.5539/mas.v9n4p160 Linear Electron Accelerators Aimed at Environmental Protection Novozhilov A.E., Filatov A.N., Shilov V.K. Biosciences Biotechnology Research Asia, September 2015. Vol. 12(Spl. Edn. 2), p. 261-268 DOI: http://dx.doi.org/10.13050/bbra/2199

Compact Accelerating System for a Multichannel Electron Linear Accelerator Novozhilov A.E., Filatov A.N., Shilov V.K. Research Journal of Pharmaceutical, Biological and Chemical Sciences (ISSN09758585-India-Scopus) July - August 2016 RJPBCS 7(5) Page No. 939-944

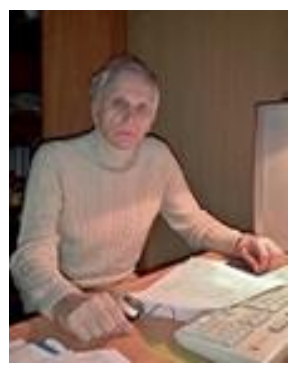

Problems of Beam Focusing by Electromagnetic Field in Linear Electron Accelerator with Standing Wave, Based on Biperiodic Structure Novozhilov A.E., Filatov A.N., Shilov V.K.Modern Applied Science; Vol. 9, No. 4; 2015: 160-169 ISSN 1913-1844 E-ISSN 19131852 Published by Canadian Center of Science and Education DOI:10.5539/mas.v9n4p160

Linear Electron Accelerators Aimed at Environmental Protection Novozhilov A.E., Filatov A.N., Shilov V.K. Biosciences Biotechnology Research Asia, September 2015. Vol. 12(Spl. Edn. 2), p. 261-268 DOI: http://dx.doi.org/10.13050/bbra/2199

Compact Accelerating System for a Multichannel Electron Linear Accelerator Novozhilov A.E., Filatov A.N., Shilov V.K. Research Journal of Pharmaceutical, Biological and Chemical Sciences (ISSN09758585-India-Scopus) July - August 2016 RJPBCS 7(5) Page No. 939-944 Closed Magnetic Trap for Confining and Heating Plasma Novozhilov A.E., Filatov A.N., Shilov V.K.Biosciences Biotechnology Research Asia, April 2015, Vol. 12(1), 647-651

DOI: http://dx.doi.org/10.13005/bbra/1708

Calculation of Resonant Frequencies and Electromagnetic Fields in Resonators of Linear Accelerators for Commercial Application, Medicine and Environmental Protection Novozhilov A.E., Filatov A.N., Shilov V.K. Research Journal of Pharmaceutical, Biological and Chemical Sciences March - April 2016 RJPBCS 7(2), pp 897-905 